\title{
Diffusion on Two-Dimensional Random Walks
}

\author{
Raf Dekeyser \\ Insituut voor Theoretische Fysica, Katholieke Universiteit Leuven, B-3030 Leuven, Belgium \\ and \\ Amos Maritan ${ }^{(a)}$ and Attilio Stella ${ }^{(b)}$ \\ Dipartimento di Fisica "Galileo Galilei" dell'Università degli Studi di Padova, I-35I31 Padova, Italy \\ (Received 30 December 1986)
}

\begin{abstract}
Analysis of Monte Carlo enumerations for diffusion on the fractal structure generated by the random walk on a two-dimensional lattice allows us to predict a behavior $\langle r\rangle \sim n^{\nu}(\ln n)^{\alpha}$ with $v=0.325 \pm 0.01$ and $\alpha=0.35 \pm 0.03$. This leads to the conjecture that $v=\alpha=\frac{1}{3}$. This value of $v$, and the presence of logarithmic corrections, are strongly supported by heuristic arguments based on Flory theory and on plausible assumptions. Evidence for the validity of these assumptions and the Flory approach is coming from remarkably successful applications in related problems.

PACS numbers: $64.60 .-\mathrm{i}, 05.40 .+\mathrm{j}, 05.50 .+\mathrm{q}, 82.20 .-\mathrm{w}$
\end{abstract}

Random walks ( $\mathrm{RW}$ ) on a regular $d$-dimensional lattice have been studied extensively in the literature, ${ }^{1,2}$ and their static fractal properties are well established. Dynamical problems on random walks, ${ }^{3,4}$ however, still present some open issues. In view of the fact that free walks are perhaps the most simple example of random fractals, these questions are a challenge and a crucial test for our understanding of the whole field of fractal dynamics, which is nowadays recognized as a major topic in several research areas, such as relaxation or transport phenomena in disordered systems, or spectral properties of biopolymers.

Through the Einstein relation, ${ }^{5}$ one is able to establish the connection

$$
\bar{d}+\zeta=d_{w}
$$

between the fractal dimensionality ${ }^{6} \bar{d}$, the resistivity exponent $\zeta$ defined by

$$
\Omega(r) \sim r^{\zeta}
$$

for the resistance $\Omega$ between two points at a distance $r$ (if we put a unit resistance on all bonds of the fractal), and the diffusion dimensionality $d_{w}$, defined by $r^{d_{w}} \sim t$, if one travels over an average distance $r$ in a diffusion process after a time $t$. Numerical calculations and $\epsilon$ expansion give sufficient evidence ${ }^{3,4}$ that in $d=3$ we have $\zeta=1.5$ and $d_{w}=3.5$, but for $d=2$ there are only contradicting opinions and evidence in the literature. It has been claimed ${ }^{4}$ that in this case, since $\bar{d}=d$, the $\mathrm{RW}$ is homogeneous in space, and therefore we should have $\zeta=0$, as in the regular two-dimensional lattice. Resistivity calculations, ${ }^{3}$ on the other hand, seem to favor $\zeta=1$.

In order to solve this ambiguity, we decided to perform numerical simulations for the diffusion on such $d=2$ random walks. We generated on the square lattice
5000 random walks of 20000 steps. On each of them, we let a particle move freely 100 times during 100 diffusion steps, under the restriction that only bonds used at least once by the RW are accessible for the diffusion. For all intermediate steps $(1 \leq t \leq 100)$ we accumulated the average square distance $a_{t}=\left\langle r^{2}(t)\right\rangle$ for these diffusions, and we tried to determine $d_{w}$ by looking for an asymptotic behavior of the form

$$
a_{n} \underset{n \rightarrow \infty}{\sim} n^{2 / d_{w}} .
$$

With standard methods of series analysis on the function

$$
f(x)=\sum a_{n} x^{n},
$$

it was evident that this led to widely varying predictions, depending on the method of analysis used, in spite of the unusual length of the series. Through previous experience with long Monte Carlo-derived series, ${ }^{7}$ which in general can lead to very consistent results, we understood that we had to look for a more original analysis. In the problem for diffusion on a $d=2 \mathrm{RW}$, logarithmic corrections come almost naturally into all formulas, as we will discuss in detail later on. Therefore, we have constructed instead of (4) the functions

$$
f(x, \alpha)=\sum a_{n}(\ln n)^{-2 \alpha} x^{n},
$$

and we applied the standard Padé analysis to the logarithmic derivative of this function at $x=1$. As suggested by Adler and Privman, ${ }^{8}$ we may expect a wildly varying Padé table, except when $\alpha$ describes correctly the logarithmic correction to (3) through

$$
a_{n}=\left\langle r^{2}(n)\right\rangle \underset{n \rightarrow \infty}{\sim} n^{2 / d_{w}}(\ln n)^{2 \alpha} .
$$

We have analyzed a central block of 32 elements of the Padé table, on which we calculated the average value $\langle v\rangle$ with $v=1 / d_{w}$ and its variance $\sigma$. In Fig. 1 we have plot- 


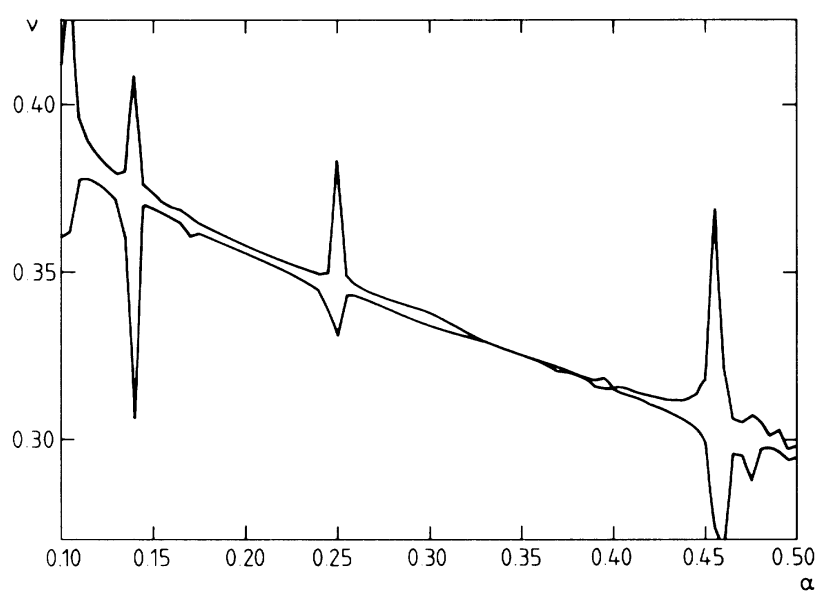

FIG. 1. Estimated boundaries for $v=1 / d_{w}$ from Padé-table analysis of the function $f(x, \alpha)$ of Eq. (5) as a function of $\alpha$. Minimum variance is clearly obtained for $\alpha \cong 0.35$, where $v \cong 0.325$.

ted the boundaries $\langle v\rangle \pm \sigma / 2$ as functions of $\alpha$. This yields us very clear estimates $v=0.325 \pm 0.010$ or $d_{w}=3.0 \pm 0.1$ and $\alpha=0.35 \pm 0.03$. This would mean that $\zeta=1$ and $\tilde{d}=2 \bar{d} / d_{w}=\frac{4}{3}$, where $\tilde{d}$ is the spectral dimension of the RW. ${ }^{9}$ This analysis suggests, as the most probable expression in the limit $t \rightarrow \infty,\left\langle r^{2}(t)\right\rangle$ $\sim(t \ln t)^{2 / 3}$.

It is worth remarking that the power-law behavior of our analysis is consistent with the extrapolation formula $\tilde{d}=8 /(4+d)$ conjectured by Banavar and Willemsen, ${ }^{10}$ and shown by them to be valid down to $d=3$. Our result shows that this formula is still valid in $d=2$, in spite of a difference in the model (in Ref. 10, conductivities of bonds were proportional to the number of visits of these bonds by the $\mathrm{RW}$ ); obviously there is universality with respect to some details of the model.

In order to understand, at least intuitively, ${ }^{11}$ our result, we now present a heuristic argument, proceeding in several steps. We will try to justify each step by testing its predictions for other related problems.

In a first step, we propose an extension to fractals of the well established Flory argument ${ }^{12}$ for the Hausdorff dimension of a self-avoiding walk on regular lattices. The repulsive energy for a walk of $N$ steps stretching over a distance $r$ in a space with fractal dimension $\bar{d}$ is easily estimated as $U \sim N^{2} r^{-d}$, whereas the entropy is, as usual, determined from the assumption of a Gaussian distribution for the distance $r$ traveled by a random walk after $N \sim r^{d_{w}}$ steps: $S \sim-r^{2} N^{-2 / d_{w}}$. Minimizing the free energy $F=U-S$ with respect to $r$ immediately yields $N \sim r^{d_{\text {saw }}}$, where

$$
d_{\mathrm{SAW}}=\frac{1}{2}(2+\bar{d}) /\left(1+d_{w}^{-1}\right) .
$$

This formula, of course, reduces to the usual Flory result $(d+2) / 3$ for regular lattices, where $d_{w}=2$. In other ap-
TABLE I. Exact and predicted values for $d_{\text {SAw }}$ on the $d=2$ Sierpinsky gaskets, for several values of the dilatation factor $b$.

\begin{tabular}{|c|c|c|}
\hline \multirow[b]{2}{*}{$b$} & \multicolumn{2}{|c|}{$d_{\mathrm{SAW}}$} \\
\hline & (Exact) & (Flory) \\
\hline 2 & 1.2522 & 1.2529 \\
\hline 3 & 1.2600 & 1.2694 \\
\hline 4 & 1.2684 & 1.2800 \\
\hline 5 & 1.2755 & 1.2876 \\
\hline 6 & 1.2815 & 1.2933 \\
\hline 7 & 1.2872 & 1.2979 \\
\hline 8 & 1.2913 & 1.3015 \\
\hline
\end{tabular}

proaches, ${ }^{13}$ the Gaussian probability distribution, used for deriving the entropic part of the free energy, was replaced by a stretched Gaussian, in order to satisfy the property that $d_{\mathrm{SAw}} / \bar{d}$ should be independent of $\bar{d}$. This property is clearly not present in our Eq. (7). The assumptions of Ref. 13 are presumably appropriate within a specific class of fractals, obtained by suitable deformations from a starting fractal. We are confident that our result covers in a satisfactory way, even if not completely, many different classes.

An immediate check of the validity of our result (7) can be made by applying it to the Sierpinsky gaskets, where exact results are available for $\bar{d}, d_{w}$, and $d_{\text {SAw. In }}$ Table I, we compare the exact values for $d_{\text {SAw }}$ with our predictions from (7), using the exact $\bar{d}$ and $d_{w}$, for gasket constructions with different dilatation factors $b .{ }^{14,15}$ The predictions seem to be correct within less than $1 \%$. Another application can be made for the SAW on the infinite incipient cluster at the percolation threshold in $d=2$. For the backbone of this fractal, $\bar{d}_{B}$ and $\tilde{d}_{B}$ have been obtained numerically, ${ }^{16}$ from which $d_{w}=2 \bar{d}_{B} / \tilde{d}_{B}$ may be derived. Our prediction from (7) is then $d_{\text {SAW }}$ $\cong 1.29$, which should be compared with the numerical estimate ${ }^{17} d_{\mathrm{SAW}} \cong 1.30$.

In all these cases, we see that our extended Flory argument leads to very accurate predictions. This gives us some confidence for its general validity, at least in problems for which $\bar{d}$ is close to $d$. Applications to problems in $d=3$ seem to lead to somewhat larger deviations (a few percentage), which, however, are of the usual quality of the Flory equations in $d=3$.

Applying (7) to the case of the RW in $d=2$, where $\bar{d}=2$, we immediately obtain

$$
N_{\mathrm{SAW}} \sim r^{2 /\left(1+d_{w}^{-1}\right)}
$$

which we believe to be valid up to logarithmic corrections.

Next, we reanalyze the Einstein relation $\sigma \sim n D$, leading to (1). The density $n$ of the $d=2 \mathrm{RW}$ is proportional to $(\ln r)^{-1}$, whereas the diffusion constant $D$ is given by

$$
D=d\left\langle r^{2}(t)\right\rangle / d t \sim r^{2-d_{w}}(\ln r)^{\alpha d_{w}},
$$


if we use (6). For the resistance $\Omega(r)$ between two points at a distance $r$ in $d=2$, we may then write

$$
\Omega(r) \sim \sigma^{-1} \ln r \sim r^{d_{w}-2}(\ln r)^{2-\alpha d_{w}} .
$$

This shows the logarithmic corrections to Eqs. (1) and (2).

As a final step in our argument, we propose the following picture for the resistance $\Omega$ on the $d=2 \mathrm{RW}$ fractal. Since the fractal is almost space filling $(\bar{d}=d)$, there may be many paths on the fractal that link two points $A$ and $B$ at a distance $r$ from each other. If we delete all parts of the fractal that do not contribute to the resistance between $A$ and $B$ (like bonds joining equalpotential points, when a voltage is applied between $A$ and $B$ ), we most probably will be left with a parallel set of essentially (topologically) one-dimensional paths of quasi SAW nature. Indeed, in contrast to a regular lattice, where electrical current between two points can flow through many paths of average length $r$, in our case the current must follow paths that were originally traced out by the RW. Stripping such random-walk paths of all pieces that cannot carry the current, we should on the average have paths of a SAW length, as described by (8). The number of these parallel paths will be proportional to the total number of bonds $\left(\sim r^{d}\right)$ divided by the length of the paths, or $r^{2} N_{\mathrm{SA} W}^{-1}$. For the total resistance, we then find

$$
\Omega(r) \sim N_{\text {SAW }}^{2} r^{-2} \text {. }
$$

Comparing this with Eq. (9) for the same quantity, we immediately find $d_{w}=3$, which corresponds to the result of our numerical simulation. Furthermore, it is possible to show, within the same calculational scheme, that logarithmic corrections may be present, although we can hardly expect our intuitive Flory arguments to predict the exact exponent $\alpha$ of the logarithmic factor in a formula like (6).

As further confirmation of the validity of our resistivity argument, leading to (10), we have applied the same formula also to the cluster of the percolation threshold in $d=2$. Using the values mentioned above ${ }^{16}$ we find in this case for the $\Omega$ exponent $\zeta=2 d_{\mathrm{SAW}}-\bar{d}_{B} \cong 1.01$. This value should be compared with independent estimates $\zeta=0.97$ (Ref. 16) and $\zeta=1.01 \pm 0.03$. ${ }^{18}$ In this way, the two important ingredients of our explanation for the $d_{w}$ on the random walk in $d=2$, namely the generalization of the Flory argument and the model for estimating the resistance $\Omega(r)$, are independently corroborated by their success on other, hopefully not too dissimilar, $d=2$ fractals.

To conclude, we believe that we have given sufficient numerical evidence and a satisfactory explanation for a value $d_{w}=3$ for the diffusion on a random-walk fractal in $d=2$. The spectral dimension $\tilde{d}=\frac{4}{3}$ turns out to coincide with the conjectured universal value for percolation clusters in any dimension. ${ }^{9}$ The above results teach us that diffusion on a space-filling random fractal can be anomalous, contrary to what one could guess at first site. To our knowledge, this is the first time that strong evidence for logarithmic corrections is produced for a problem in fractal dynamics. We believe this to be a remarkable feature, which, moreove, explains the previous difficulties in determining the spectral properties of the random walk in $d=2$.

\footnotetext{
(a) Also at Istituto Nazionale di Fisica Nucleare, Sezione di Padova, Italy.

(b) Also at International School for Advanced Studies, I34014 Trieste, Italy, and Unità di Padova, Gruppo Nazionale di Struttura della Materia del Consiglio Nazionale delle Ricerche e Centro Interuniversitario di Struttura della Materia, Padova, Italy.
}

${ }^{1}$ E. W. Montroll and G. H. Weiss, J. Math. Phys. 6, 167 (1965).

${ }^{2}$ K. J. Falconer, The Geometry of Fractal Sets (Cambridge Univ. Press, Cambridge, MA, 1985).

${ }^{3}$ J. R. Banavar, A. B. Harris, and J. Koplik, Phys. Rev. Lett. 51, $1115(1983)$

${ }^{4}$ S. Havlin, G. H. Weiss, D. Ben-Avraham, and D. Movshovitz, J. Phys. A 17, L849 (1984).

${ }^{5}$ Y. Gefen, A. Aharony, and S. Alexander, Phys. Rev. Lett. 50, 77 (1983).

${ }^{6}$ B. B. Mandelbrot, The Fractal Geometry of Nature (Freeman, San Francisco, 1982)

${ }^{7}$ A. L. Stella, R. Dekeyser, and A. Maritan, in Fractals in Physics, edited by L. Pietronero and E. Tosatti (Elsevier, Amsterdam, 1986).

${ }^{8}$ J. Adler and V. Privman, J. Phys. A 14, L463 (1981).

${ }^{9} \mathrm{~S}$. Alexander and R. Orbach, J. Phys. (Paris), Lett. 43, L625 (1982); R. Rammal and G. Toulouse, J. Phys. (Paris), Lett. 44, L13 (1983).

${ }^{10}$ J. R. Banavar and J. F. Willemsen, Phys. Rev. Lett. 53, 1609 (1984).

${ }^{11}$ At first sight, this result could even seem to go against intuition. Some authors indeed claimed [see Ref. 4 and J. S. Helman, A. Coniglio, and C. Tsallis, Phys. Rev. Lett. 53, 1195 (1984)] that the diffusion on a space-filling fractal like the RW in $d=2$, should be normal, i.e., $d_{w}=2$.

${ }^{12} \mathrm{P}$. G. de Gennes, Scaling Concepts in Polymer Physics (Cornell Univ. Press, Ithaca, NY, 1979).

${ }^{13}$ J. Vannimenus, J. Phys. (Paris), Lett. 45, L1071 (1984)

${ }^{14}$ S. Elezović, M. Knežević, and S. Milošević, unpublished

${ }^{15}$ R. Hilfer and A. Blumen, J. Phys. A 17, L537 (1984).

${ }^{16}$ L. de Arcangelis, S. Redner, and A. Coniglio, Phys. Rev. B 31, 4725 (1985).

${ }^{17}$ K. Kremmer, Z. Phys. B 45, 2679 (1981)

${ }^{18}$ L. Puech and R. Rammal, J. Phys. C 16, L.1197 (1983). 